Document downloaded from:

http://hdl.handle.net/10251/176992

This paper must be cited as:

Alcántara-Ávila, F.; Gandía-Barberá, S.; Hoyas, S. (2019). Evidences of persisting thermal structures in Couette flows. International Journal of Heat and Fluid Flow. 76:287-295. https://doi.org/10.1016/j.ijheatfluidflow.2019.03.001

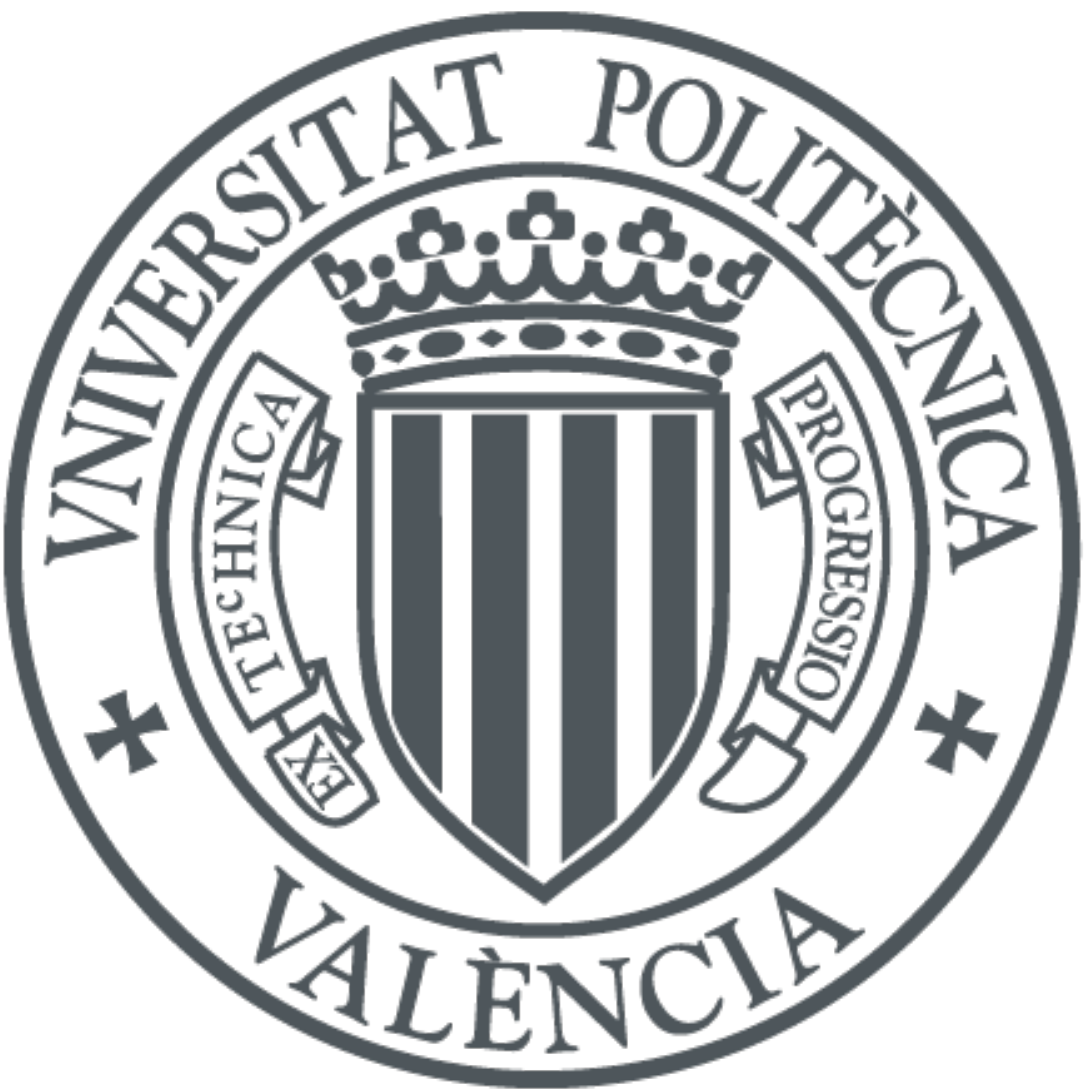

The final publication is available at

https://doi.org/10.1016/j.ijheatfluidflow.2019.03.001

Copyright Elsevier

Additional Information 
1

2

3 Francisco Alcántara-Ávilaa , Sergio Gandía-Barberáa, Sergio Hoyasa,**

$4 \quad{ }^{a}$ Instituto Universitario de Matemática Pura y Aplicada, Universitat Politècnica de

5

\section{Evidences of persisting thermal structures in Couette flows}

\author{
València, Valencia 46022, Spain.
}

\begin{abstract}
DNS of passive thermal turbulent Couette flow at several friction Reynolds numbers (180, 250, and 500), and the Prandtl number of air are presented. The time averaged thermal flow shows the existence of long and wide thermal structures never described before in Couette flows. These thermal structures, named CTFS (Couette Thermal Flow Superstructures), are defined as coherent regions of hot and cold temperature fluctuations. They are intrinsically linked to the velocity structures present in Couette flows. Two different 2D symmetries can be recognized, which get stronger with the Reynolds number. These structures do not affect the mean flow or mean quantities as the Nusselt number. However, turbulent intensities and thermal fluxes depend on the width of the structures, mainly far from the walls. Since the width of the structures is related to the channel width, the statistics of thermal Couette flow are to some point box-dependent.
\end{abstract}

\section{Introduction}

Wall turbulence is probably one of the open problems in physics with more applications in daily life. Due to the highly non-linear behaviour of

*Corresponding author. e-mail: serhocal@mot.upv.es. Tel: +34 - 963877007 (ext Toresint submitted to Elsevier

July 4, 2019 
wall-turbulent flows, mainly basic research is restricted to simplified geometries, as can be turbulent channel flows or boundary layers. In turbulent channels, the fluid is confined between two parallel plates and the flow is driven either by pressure, Poiseuille flows (PFs) [1, 2, 3, 4, 15, or shear, Couette flows $(\mathrm{CFs})[6,7,8,9$. Until very recently, CFs have been simulated at a relatively small Reynolds number [6, 7, 9, 10, 11]. This is due to the existence of very large-scale roll-like motions extending along the domain, which were found experimentally [12, 13, 14]. In the later, Kitoh and Umeki used an experimental apparatus with size $5120 \times 27 \times 880 \mathrm{~mm}$ with a lower wall moving-belt. They performed several experiments at $R e=U_{w} h / \nu=3750$, where $U_{w}$ was the half of the velocity of the moving-belt; $h$, the semi-height of the channel; and $v$, the kinematic viscosity; using a hot-wire probe to measure flow statistics. In order to reduce the randomness of the appearance of the structures in the experiment, they used a vortex generator in the core region of the channel. The streamwise rolls that appeared downstream this vortex generator became quasistationary without a noticeable decay. In order to properly capture these rolls in numerical simulations, long and wide computational boxes are needed, which makes simulations only available for the largest supercomputers. In isothermal flows, these rolls are defined as coherent regions of either positive or negative streamwise velocity fluctuations. They appear in pairs, creating a structure in the velocity field composed by a couple of counter-rotating vortices aligned with the streamwise direction [7, 15]. There is not a clear criterion to identify these structures. In ducts, this structures has been identfied using threshold functions [? ], but this has still to be done in Couette flows. They are mostly identified through 


\begin{tabular}{ccccccc} 
Work & $R e_{\tau}$ & $L_{x} / \pi$ & $L_{z} / \pi$ & $\Delta x^{+}$ & $\Delta y^{+}$ & $\Delta z^{+}$ \\
\hline & 52 & 14 & 4 & 4.54 & $0.07-2.33$ & 2.6 \\
Tsukahara et al. [6] & 126 & & & 11.03 & $0.18-5.67$ & 6.31 \\
& 126 & 20 & 2 & 7.88 & $0.18-5.67$ & 5.90 \\
Avsarkisov et al. [7] & $125-550$ & 20 & 6 & 13 & $0.92-5.9$ & 6.5 \\
Pirozzoli et al. [8] & $171-986$ & 18 & 8 & 7 & $0.08-8.8$ & 5 \\
Lee and Moser [9] & $93-501$ & 100 & 5 & 10.25 & $0.040-6.33$ & 5.13 \\
Kraheberger et al. [16] & 547 & 60 & 6 & 12.58 & $0.041-6.71$ & 5.03 \\
Gandía-Barberá et al. [10] & $250-1000$ & 8 & 3 & 12.2 & $0.42-7.2$ & 6.13 \\
Komminaho et al. [11] & 132 & 128 & 6 & 9.4 & $0.83-2.3$ & 4.7 \\
Kawamura et al. [17] & 52 & 28 & 8 & 13.5 & $1.9($ mean) & 7.7 \\
Debusschere and Rutland [18] & 180,395 & 2 & 1 & 9.88 & $0.44-13$ & 4.94 \\
Tsukahara et al. [19] & 160 & 3.8 & 0.6 & 8.3 & 1.6 & 5 \\
Present work & 52,126 & 28.5 & 4 & 11.0 & $2.6(\mathbf{m e a n})$ & 6.3 \\
\hline
\end{tabular}

Table 1: Parameters of previous CF simulations. Works in bold denotes that the thermal field has also been simulated. Third and fourth column show the maximum computational domain used in each work in $x$ and $z$, respectively. Second column shows the ranges of $R e_{\tau}$ simulated at the corresponding computational boxes. Last three columns show the mesh resolution for each highest $R e_{\tau}$ case.

visualization employing some filtering [7, 9]. As it is shown later, there is a clear organization of the velocity and thermal rolls in larger structures, with two clear symmetries. As these rolls and structures have not received a name, the generic term CTFS (Couette Thermal Flow Superstructure) will set composed by several of them.

Focusing in turbulent thermal flows, in a recent study for NASA, Slotnick et al. [20] highlighted the importance of thermal flows in aeronautical applications in the foreseeable future. To cite another example, for Prandtl numbers different to the one of air, a better knowledge of the dynamics of thermal flows is needed for the simulation of nuclear Liquid Metal Reactors 
(LMR) [21, 22]. Finally, heat transmission will be critical in the new transportation system Hyperloop, as the flow between the pod and the pipe can be thought as a CF. There exists a rich bibliography about the problem of heat transfer in PFs, see [23, 24] and references therein. However, up to the knowledge of the authors, only a few works address the problem of heat transfer for CFs [18, 17, 19]. These works were a challenge in their times, but unfortunately in most of them, either the computational box used is too narrow and short to properly describe the CTFS, or the Reynolds number used is low. In fact, in the work of Debusschere and Rutland [18] it is explicitly stated that they are using a narrow box to remove the effects of the velocity rolls. These authors affirm that these rolls are not probably present in real-life devices. However, as it is said before, the rolls have been found experimentally [12, 13, 14]. They are very long and could be even infinite in length [9]. The rolls are also stable even to transversal flows [16] and they affect the flow as secondary flows in ducts do [25]. Moreover, as it is stated in this paper, CTFS do affect both the statistics of the flow and the thermal field, as it is composed by rolls of both of fields. This appears to be an effect of Couette boundary conditions. Table 1 shows the parameters of previous CFs simulations, such as $R e_{\tau}$, computational box size and mesh resolution. In the case of a PF, Lozano-Durán and Jiménez for the flow field [26], and Lluesma et al. for thermal flows [27], found out that relatively small computational box of stream- and span-wise sizes of only $2 \pi h \times \pi h$ can satisfactorily recover the one-point statistics of the flow. The existence and characterization of the CTFS, and its influence in the one-point statistics is addressed for the first time in this work. 


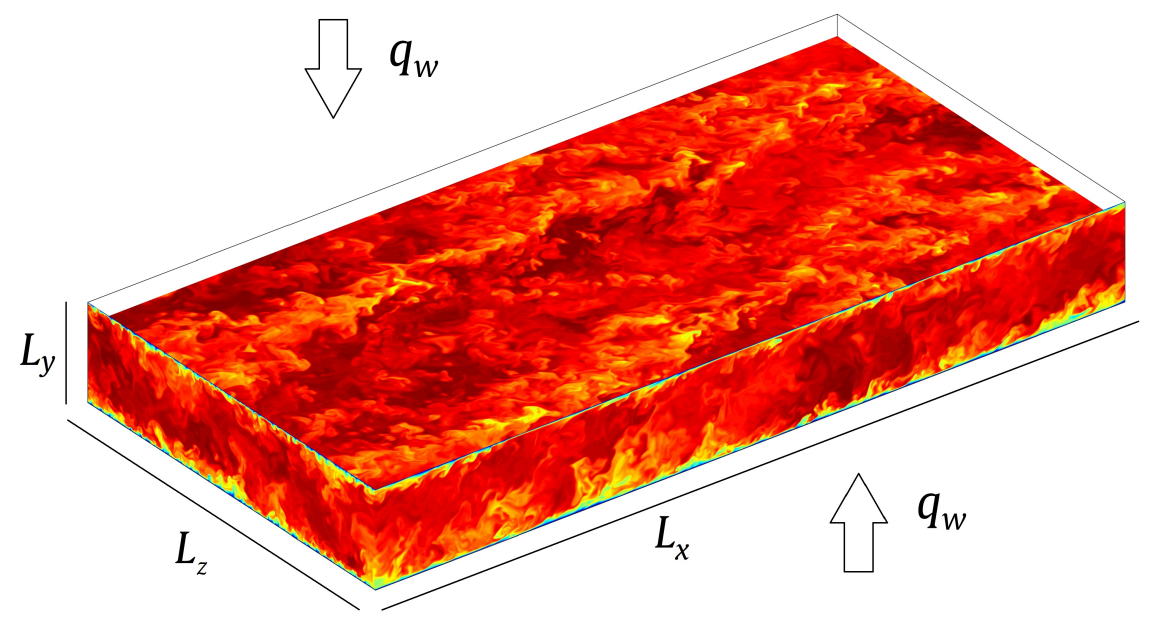

Figure 1: Thermal flow at $R e_{\tau}=500$ for $\operatorname{Pr}=0.71$. The flow is driven by the shear of the top wall and moves from the left to the right. Both walls are considered isothermal, and the thermal heat flux, $q_{w}$, is constant.

The structure of the paper is as follows. In the second section, the numerical method is presented and validated. In the third section, the influence of the CTFS in the mean flow and in the one-point statistics is accounted and discussed. Finally, the fourth section contains the summary and conclusions.

\section{Numerical setup}

To perform this study, two DNS of a passive thermal flow in a Couette turbulent channel at $R e_{\tau}=250$ and 500, and $\operatorname{Pr}=0.71$ have been conducted within a computational box of $L_{x}=16 \pi h, L_{y}=2 h$ and $L_{z}=6 \pi h$, with periodicity in the streamwise and spanwise directions. These are the cases $\mathrm{V}$ and VI in Table 2. A schematic representation of the computational box is shown in Figure 1, where the thermal field is plotted. Here, $R e_{\tau}=u_{\tau} h / \nu$ is the friction Reynolds number, $\operatorname{Pr}$ is the molecular Prandtl number, $u_{\tau}$ is the friction velocity, $h$ is the semi-height of the channel and $\nu$ is the kinematic 


\begin{tabular}{|c|c|c|c|c|c|c|c|c|}
\hline Cases & Line & $R e_{\tau}$ & $R e$ & Box & $N_{x}$ & $N_{y}$ & $N_{z}$ & $T u_{\tau} / h$ \\
\hline 1 & 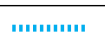 & 166 & 3500 & $4 \pi h \times 0.5 \pi h$ & 384 & 201 & 144 & 57.3 \\
\hline II & $\cdots$ & 177 & 3500 & $2 \pi h \times \pi h$ & 192 & 201 & 192 & 79.9 \\
\hline III & - & 176 & 3500 & $4 \pi h \times 2 \pi h$ & 384 & 201 & 384 & 39.2 \\
\hline IV & --- & 178 & 3500 & $16 \pi h \times 6 \pi h$ & 1536 & 251 & 1152 & 59.1 \\
\hline V & . & 262 & 5400 & $16 \pi h \times 6 \pi h$ & 1536 & 251 & 1152 & 88.9 \\
\hline VI & - & 476 & 10500 & $16 \pi h \times 6 \pi h$ & 3072 & 251 & 2304 & 58.1 \\
\hline
\end{tabular}

Table 2: Parameters of the simulation. Friction and bulk Reynolds numbers are shown in columns three and four, respectively. The fifth column shows the box size of each case. $N_{x}, N_{y}$ and $N_{z}$ are the number of collocation points in each direction. The last column contains the time spent to compile statistics in terms of eddy turnovers. The lines used through the paper to identify the different cases are shown in the second column.

viscosity. In addition, four CF simulations with different size of boxes in $x$ and $z$ dimensions ( $4 \pi h \times 0.5 \pi h, 2 \pi h \times \pi h, 4 \pi h \times 2 \pi h$ and $16 \pi h \times 6 \pi h$ ) were also conducted at $R e_{\tau}=180$ and the Prandtl number of air (cases I-IV in Table 2). These simulations were performed to check whether or not the size of the box has an influence in the rolls and, consequently, in the mean and one-point statistics. The superscript $(+)$ indicates that the quantities have been normalized either by $u_{\tau}$ or by the friction temperature $\theta_{\tau}$. The streamwise, wall-normal, and spanwise coordinates are $x, y$, and $z$. The corresponding velocity components are $U, V$ and $W$ or, using index notation, $U_{i}$. Defining the average operator $\langle\cdot\rangle_{x_{i}}$ as

$$
\langle\phi\rangle_{x_{i}}=\frac{1}{L_{x_{i}}\left(t_{1}-t_{0}\right)} \int_{t_{0}}^{t_{1}} \int_{0}^{L_{x_{i}}} \phi d x_{i} d t
$$

the value of $\langle\phi\rangle_{x}$ can be thought as the mean in $x$ of the time-averaged field $\phi$. Statistically averaged quantities in $x$ and $z$ are denoted by an overbar, $\bar{\phi}=\langle\phi\rangle_{x z}$, whereas fluctuating quantities are denoted by lowercase letters, i. e., $U=\langle U\rangle_{x z}+u=\bar{U}+u$. 
The temperature is simulated as a passive scalar and denoted by $T$. The transformed temperature is $\Theta$, which is defined as $\Theta=T-T_{w}$, where $T_{w}$ is the temperature at the wall. In the streamwise direction, it is assumed that temperature increases linearly. Subtracting $T_{w}$ to $T$ makes $\Theta$ periodic, as $T_{w}$ carries the non-periodic part of the thermal flow. These conditions are known as Mixed Boundary Conditions (MBC) [28]. Among other things, MBC allow the use of highly efficient Fourier methods in the streamwise direction.

The flow can be described by means of the momentum and mass balance equations, as well as the energy conservation principle. These equations are later solved using the LISO code, which has successfully been employed to run some of the largest simulations of turbulence [2, 7, 10, 24, 27, 29, 30]. Briefly, the code uses the same strategy than [1], but using a seven-point compact finite differences in the wall-normal direction with fourth-order consistency and extended spectral-like resolution 31. The temporal discretization is a third-order semi-implicit Runge-Kutta scheme [32]. The wall-normal grid spacing is adjusted to keep the resolution at $\Delta y=1.5 \eta$, i.e., approximately constant in terms of the local isotropic Kolmogorov scale $\eta=\left(\nu^{3} / \epsilon\right)^{1 / 4}$. In wall units, $\Delta y^{+}$varies from 0.83 at the wall, up to $\Delta y^{+} \simeq 2.3$ at the centerline. The wall-parallel resolution in physical space for $x$ and $z$ is $\Delta x^{+} \simeq$ 8.4 and $\Delta z^{+} \simeq 4.3$

In every simulation, the flow had to evolve from an initial file, which has been taken from previous different simulations. The code was run until the transition phase had passed and the flow had adjusted to the new set of parameters. Once the flow is in a statistically steady state, statistics are 
compiled. The running times used to compile statistics are shown in the rightmost column of Table 2. They are given in terms of eddy-turnovers. The transitions before the simulations reached a statistically steady state, which can be very time consuming, are not contemplated in this table.

In order to further validate the database, two procedures have been followed. First, the total shear stress equation, which in CFs is

$$
1+\frac{d \bar{U}}{d y}+\overline{u v}=0
$$

is used to validate the isothermal flow. Second, the total heat flux is calculated and compared with the molecular and turbulent heat flux. The flux conservation equation is given by [24]

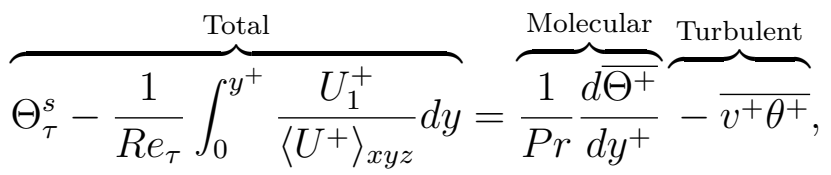

where $\Theta_{\tau}^{s}=\left.\partial_{y} \Theta\right|_{y=0} /\left(R e_{\tau} \operatorname{Pr}\right)$ is the friction temperature at the stationary wall.

The results of this validation are shown in Figure 2, for cases V and VI. In Figure 2a, apart from the small error, a very good agreement is found between both simulations, as the curves collapse almost perfectly. Molecular and turbulent heat fluxes are compared with the total flux in Figure $2 \mathrm{~b}$. The total error is defined as the difference between the RHS and the LHS of Equation 3. It has been considered that enough statistics were obtained when this error was below $10^{-3}$. 


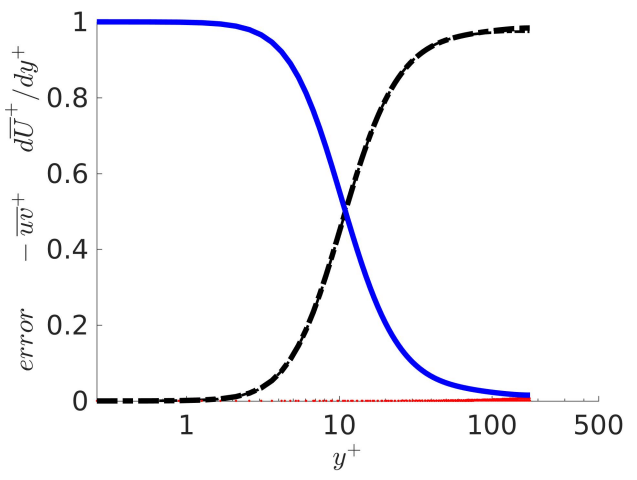

(a)

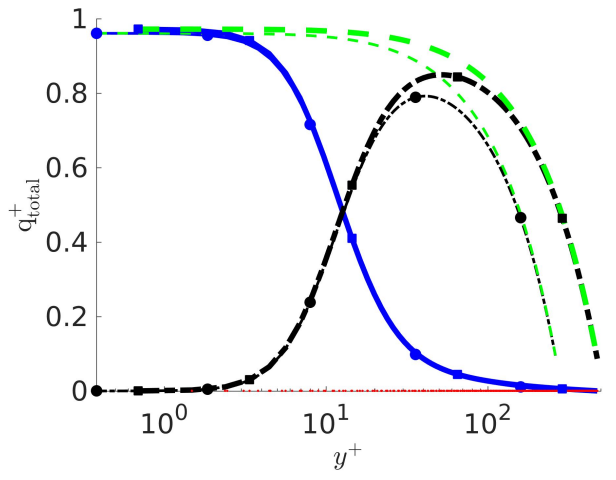

(b)

Figure 2: Colour online. For $R e_{\tau}=250$ (thin lines) and 500 (thick lines). (a) Shear stress: $d \bar{U}^{+} / d y^{+}$(blue, solid), $\overline{u v}^{+}$(black, dash-dot) (b) Heat fluxes: Molecular (blue, solid), turbulent (black, dash-dot) and total (green, dash). In both cases, the red pointed line represents the difference between LHS and RHS of either Equation 2 or 3

\section{Results and discussion}

CFs and PFs affect in different ways the thermal flow under MBC for these low Reynolds numbers. A first result is that mean and one-point statistics are no longer symmetric. In Figure 3a, it can be seen that temperature profiles are slightly different in both halves of the channel for CFs. On the other hand, the PF thermal profile is perfectly symmetric [27]. A first insight of the collapse of the statistics depending on the computational box size can be obtained from Figure $3 \mathrm{~b}$. Here, temperature profiles of the bottom part of the channel of cases I-IV are shown. All profiles of $\overline{\Theta^{+}}$collapse fairly well, except for case I. This case uses a computational box similar to the one in [18]. The collapse error is probably due to the narrowness of the box [27]. Another remarkable difference between CFs and PFs is the presence or absence of a thermal logarithmic layer between the viscous and the outer layers. For PFs, this logarithmic layer appears for $\operatorname{Pr} \approx 0.3$ and above [24]. However, for CFs, 


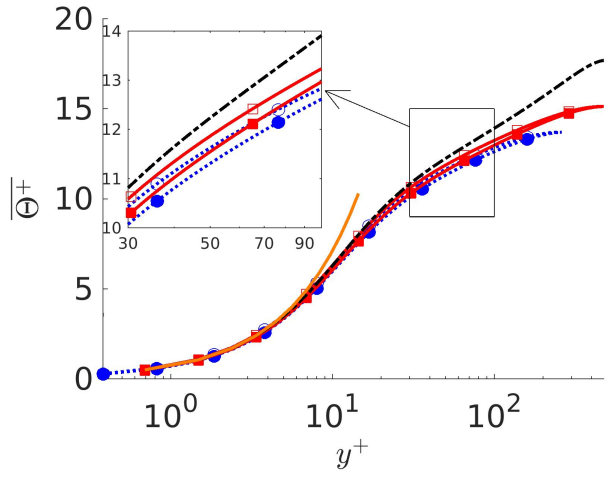

(a)

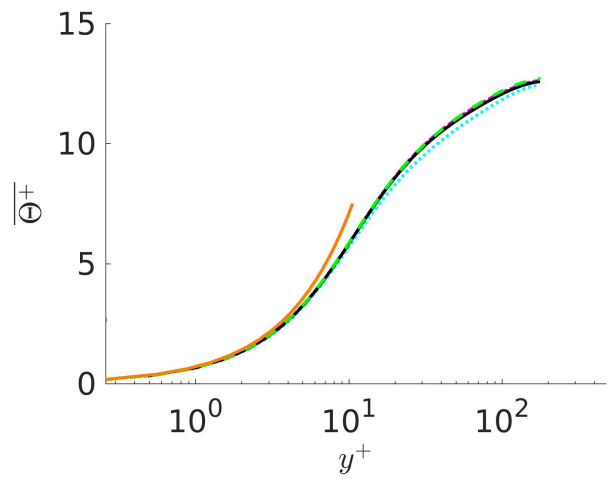

(b)

Figure 3: Colour online. (a) $\overline{\Theta^{+}}$for cases V (blue dashed) and VI (red solid). Closed symbols, bottom half. Empty symbols, top half. Black dash-pointed line represents a PF at $R e_{\tau}=497$. (b) $\overline{\Theta^{+}}$for cases I-IV at the stationary wall. In both cases, orange solid line: thermal law of the wall, $\overline{\Theta^{+}}=\operatorname{Pr} \cdot y^{+}$. Colours as in Table 2

it is not present for $\operatorname{Pr}=0.71$. It is uncertain if this logarithmic layer in the temperature field will appear for higher Prandtl numbers in a CF. However, as it is said before, CFs are far more computationally expensive than PFs. To see if a thermal logarithmic layer does exist in CFs will still be a challenge for the next decade.

In Figure 4 turbulent intensities for the three different Reynolds numbers are shown. As a general result, it is seen that the maximum of all turbulent intensities increases and moves towards the wall with an increase of the Reynolds number. A difference between CFs and PFs appears in $u^{\prime+}$, Figure 4a. A second maximum can be observed for the higher Reynolds number, 500. This was already noted in previous simulations [7]. and from this work it is confirmed that for $R e_{\tau}=500, u^{\prime+}$ shows a second maximum in the central region of the channel. Also, as it was shown before for $\overline{\Theta^{+}}$, intensities related with temperature are not totally symmetric or antisymmetric. This 


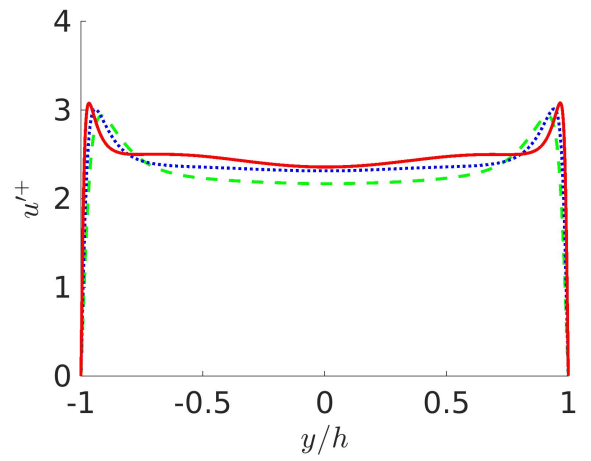

(a)

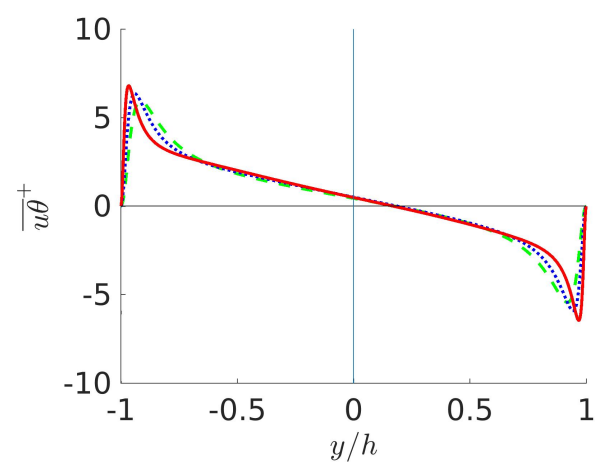

(c)

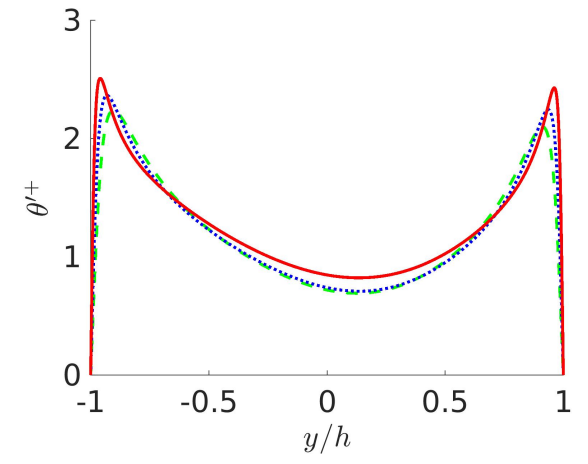

(b)

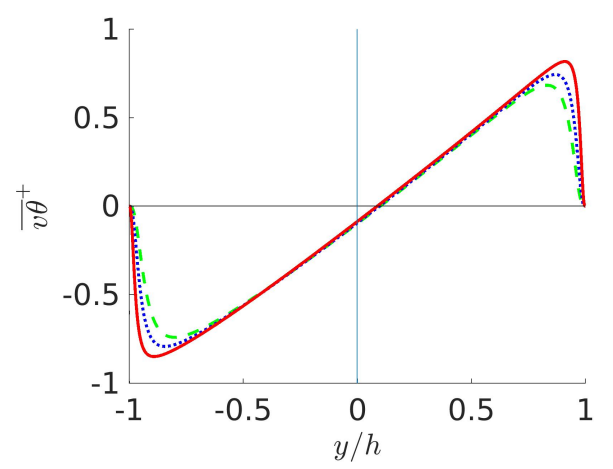

(d)

Figure 4: Colour online. (a) $u^{\prime+}$, (b) $\theta^{\prime+}$, (c) $\overline{u \theta}$ and (d) $\overline{v \theta}$ for cases IV-VI. Colours as in Table 2

can be seen in Figures $4 \mathrm{~b}, 4 \mathrm{c}$ and $4 \mathrm{~d}$. Another important point is the antisymmetry obtained for the turbulent intensity $\overline{u \theta}$, Figure $4 \mathrm{c}$. This opposes to the behaviour of $\overline{u \theta}$ in a PF when the MBC is employed, where a symetric profile is obtained. This is discussed below.

A distinctive feature of CFs is the existence of velocity large instantaneous structures in the flow [10, 11]. Corresponding long and wide structures of the thermal fluctuation field are also present. These instantaneous structures, Figure 5, appear in a strip-like pattern in $x-z$ planes. Isocontours 


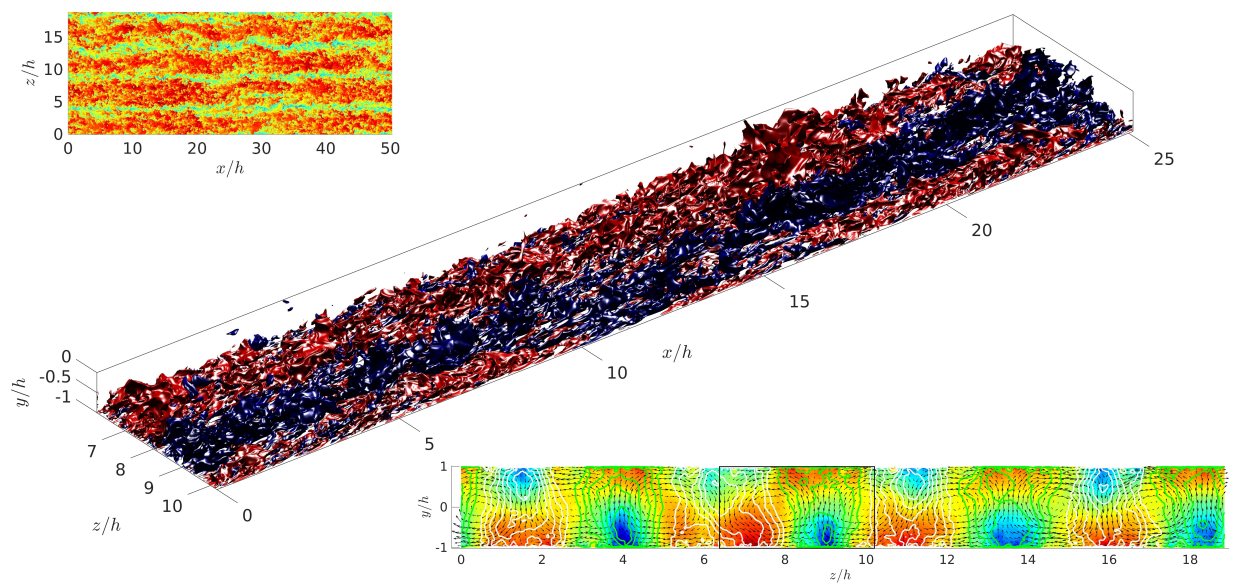

Figure 5: Instantaneous value of $\theta$ at $R e_{\tau}=500$. Top: $x-z$-plane at $y=0.3 h$. Middle: isocontours of $\theta$. Red, hotter than mean; blue, colder than mean. Bottom: mean in the streamwise direction of $u$ (isolines), $v$ and $w$ (arrows) and $\theta$ (contours). The black box indicates the region plotted in the middle figure.

of $\theta, 5$ middle, show long and wide, coherent three dimensional thermal structures, that mainly extend along the streamwise direction. CTFS are a consequence of this particular distribution of the values of $\vec{u}$ and $\theta$, as they reflect the effect of these instantaneous structures in the time-averaged flow and thermal field. CTFS are defined as a characteristic of the time-averaged perturbations, which does not need to be zero. A first proof of the existence of the CTFS appears in the $x$-averaged fields. The values of $\langle u\rangle_{x},\langle v\rangle_{x}$, $\langle w\rangle_{x}$, and $\langle\theta\rangle_{x}$ are shown in Figure 6b. In this figure, white and green lines represent contours of positive and negative $\langle u\rangle_{x}$, respectively. $\left(\langle v\rangle_{x},\langle w\rangle_{x}\right)$ vector field is represented by arrows. Colours show contours of $\langle\theta\rangle_{x}$. While the $U$-velocity structures expand across the whole height of the channel, a pair of hot-cold thermal structures appear for every velocity structure. These 
thermal structures are as long as the box itself, $L_{x}=16 \pi h$ (Figure 6c), in the same way as the velocity structures [9].

The black box of Figure 6b shows the footprint of a CTFS. These structures are made of two velocity rolls (positive-negative velocity fluctuations) and four thermal ones. They are organized in such a way that the thermal flux, $\overline{u \theta}$, is positive in the lowest part of the channel and negative in the top one. A large vortex in $\langle v\rangle$ and $\langle w\rangle$ appears at the center of the CTFS.

In PFs, these structures do not exist. In fact, the time averaged of any perturbation field is simply noise. This is due to the inclination of large-scale structures in the PF. In this context, Abe et al. [33] recently examined the effects of the streamwise domain size in the PF for a flow field for $R e_{\tau}=395$ and 1020. They found out that when the streamwise domain is reduced to $L_{x}^{+} \approx 400$ for $R e_{\tau}=1020$, the two-dimensional behavior is indeed established in the PF. Thus, only trivial symmetries are present. CTFS, on the other hand, exhibit two non-trivial symmetries never described before. First, there is a translation symmetry of period $2 L_{R}$. This length is the distance between the centers of two consecutive rolls. In this case is $L_{R} / h=6 \pi / 8 \approx 2.3$. Second, there exists a symmetry, for $\langle\theta\rangle_{x}$, with respect to the origin of every $(v, w)$ vortex (see black box of Figure 6b).

Quantities related to the mean flow seem not to be seriously affected by the CTFS. As an example, the most important parameter for modeling thermal boundary layers, the Nusselt number, has been computed as in [34,

$$
N u=\frac{L \mathfrak{h}}{\kappa}=\frac{2 R e_{\tau} P r}{\left\langle\overline{\Theta^{+}}\right\rangle_{m}}=\frac{2 P e_{\tau}}{\left\langle\overline{\Theta^{+}}\right\rangle_{m}},
$$

where $L$ is a characteristic length, in this case it is the channel height of the 


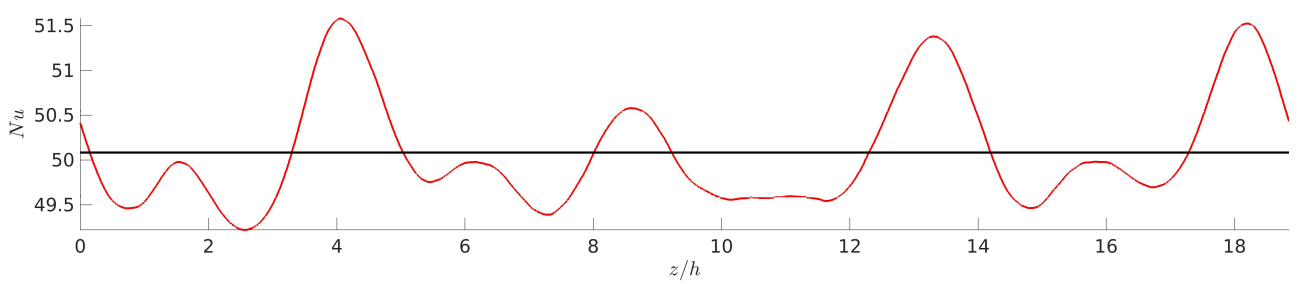

(a)

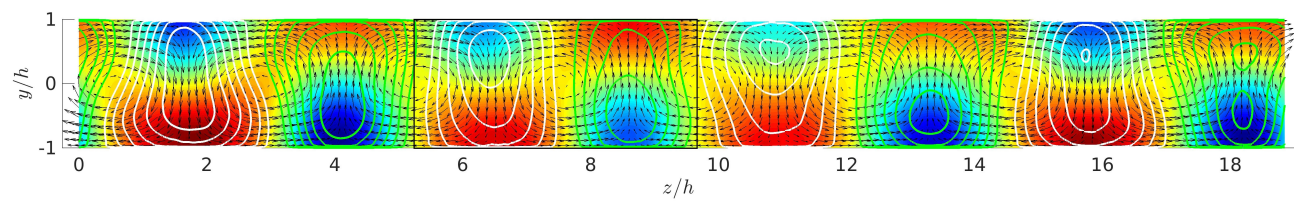

(b)

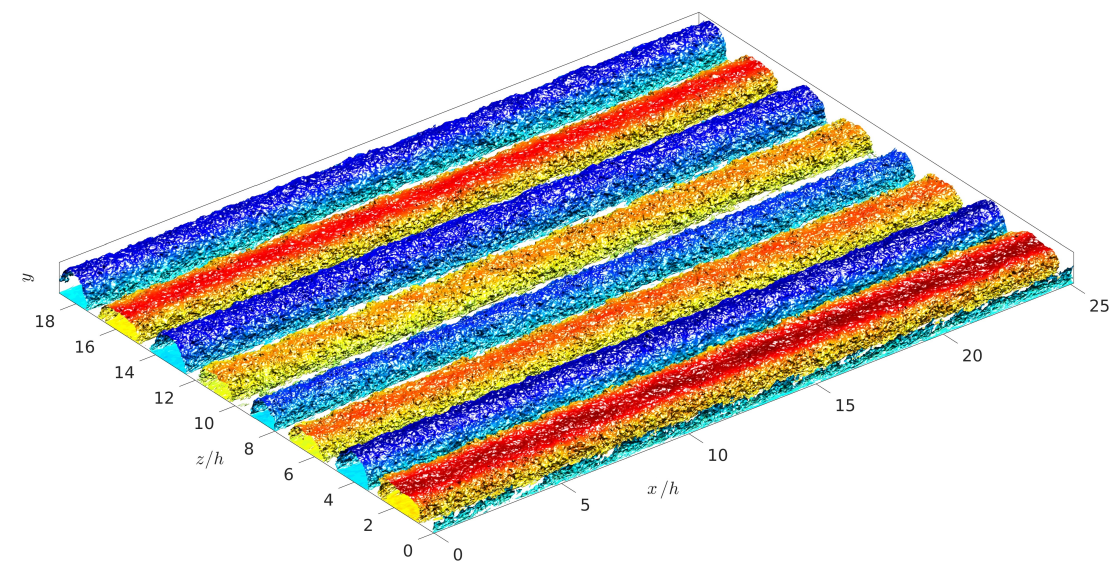

(c)

Figure 6: Colour online. Case VI. (a) Local Nusselt number averaged in $x$ direction (red) and averaged Nusselt number (black). (b) Contours of $\langle\theta\rangle_{x}$. White and green lines represent contours of positive and negative $\langle u\rangle_{x}$, respectively. $\left(\langle v\rangle_{x},\langle w\rangle_{x}\right)$ vector field is represented by arrows. (c) Isocontours of cold (blue) and hot (red) $\langle\theta\rangle$. The values of the isocontours are $0.4\langle\theta\rangle_{\min }$ and $0.4\langle\theta\rangle_{\max }$ for the cold and hot $\langle\theta\rangle$, respectively. 
computational box, $2 h ; \mathfrak{h}$ is the convective heat transfer coefficient; $\kappa$ is the thermal conductivity; and $\left\langle\overline{\Theta^{+}}\right\rangle_{m}$ is the mixed mean transformed temperature, defined as 34

$$
\left\langle\overline{\Theta^{+}}\right\rangle_{m}=\int_{0}^{1} \overline{U^{+} \Theta^{+}} d y / \int_{0}^{1} \overline{U^{+}} d y
$$

Table 3 summarizes the difference in Nusselt number for CFs and PFs. Values of $N u_{P}$ at $R e_{\tau}=180$ and 500 have been obtained from [34] and [24], respectively. In order to obtain $N u_{P}$ at $R e_{\tau}=250$ a new simulation was run by the authors and then $N u_{P}$ was computed using (5).

One can consider the hypothesis of a linear behaviour of the Nusselt number in the range from $R e_{\tau}=180$ to 500 . Therefore, precise values of $N u_{P}$ have been extrapolated to the Reynolds number of the corresponding CF. All simulations I-IV give approximately the same value (less than a $0.6 \%$ deviation) of $N u_{C}$, regardless of the box size. Thus, short and narrow boxes do not introduce a big error when calculating the Nusselt number. An interesting property to be investigated is the difference in $N u$ between CFs and PFs. As can be seen in Table 3, this difference gets reduced with an increase of $R e_{\tau}$.

\begin{tabular}{cccc}
$R e_{\tau}$ & $N u_{C}$ & $N u_{P}$ & $\Delta(\%)$ \\
\hline 178 & 21.60 & 18.33 & 17.9 \\
262 & 29.39 & 25.35 & 16.0 \\
476 & 47.96 & 42.86 & 11.9
\end{tabular}

Table 3: $N u$ for each Reynolds in a CF, second column, and a PF, third column. The fourth column shows the relative difference.

However, local Nusselt number can be different to the average one due to the effect of the CTFS. In Figure 6a local Nusselt number for Case VI is 
shown in red and the averaged one, in black. Despite it looks like hot rolls compensate the cold ones when averaging in the $y$ direction (Figure 6b), local Nusselt number shows that the lower thermal rolls are stronger, especially the cold ones. For this reason, $\langle N u\rangle_{x}$ have greater peaks above the average $N u$ in the $z$ coordinates where a cold roll is present in the lower part of the channel. Here, local $N u$ number differs up to a $3 \%$ from the averaged one. Remark that local Nusselt number shows two frequencies: one that corresponds to the wavelength of the CTFS. The other, whose amplitude is half of the CTFS one and whose intensity is lower, corresponds to smaller structures that cannot be seen in isocontours plots of mean $u^{\prime+}$ and $\theta^{\prime+}$, but that will be observed in the spectral analysis.

To conclude the analysis of the Nusselt number, a comparisson of the results obtained in this work with the correlation obtained by Abe and Antonia in [35] for PFs has been performed. This correlation expresses the Nusselt number as a function of Reynolds bulk number, $R e_{b}$, where the entire height of the channel is considered for its calculation,

$$
N u=h_{t} R e_{b} \operatorname{Pr}
$$

where $h_{t}$ is the heat transfer coefficient, computed as

$$
h_{t}=\frac{\sqrt{\frac{C_{f}}{2}}}{2.18 \ln \left(\operatorname{Re}_{b} \frac{\sqrt{C_{f}}}{2 \sqrt{2}}\right)+2.4},
$$

where $C_{f}$ is the skin friction coefficient, which is obtained form the next expression, 


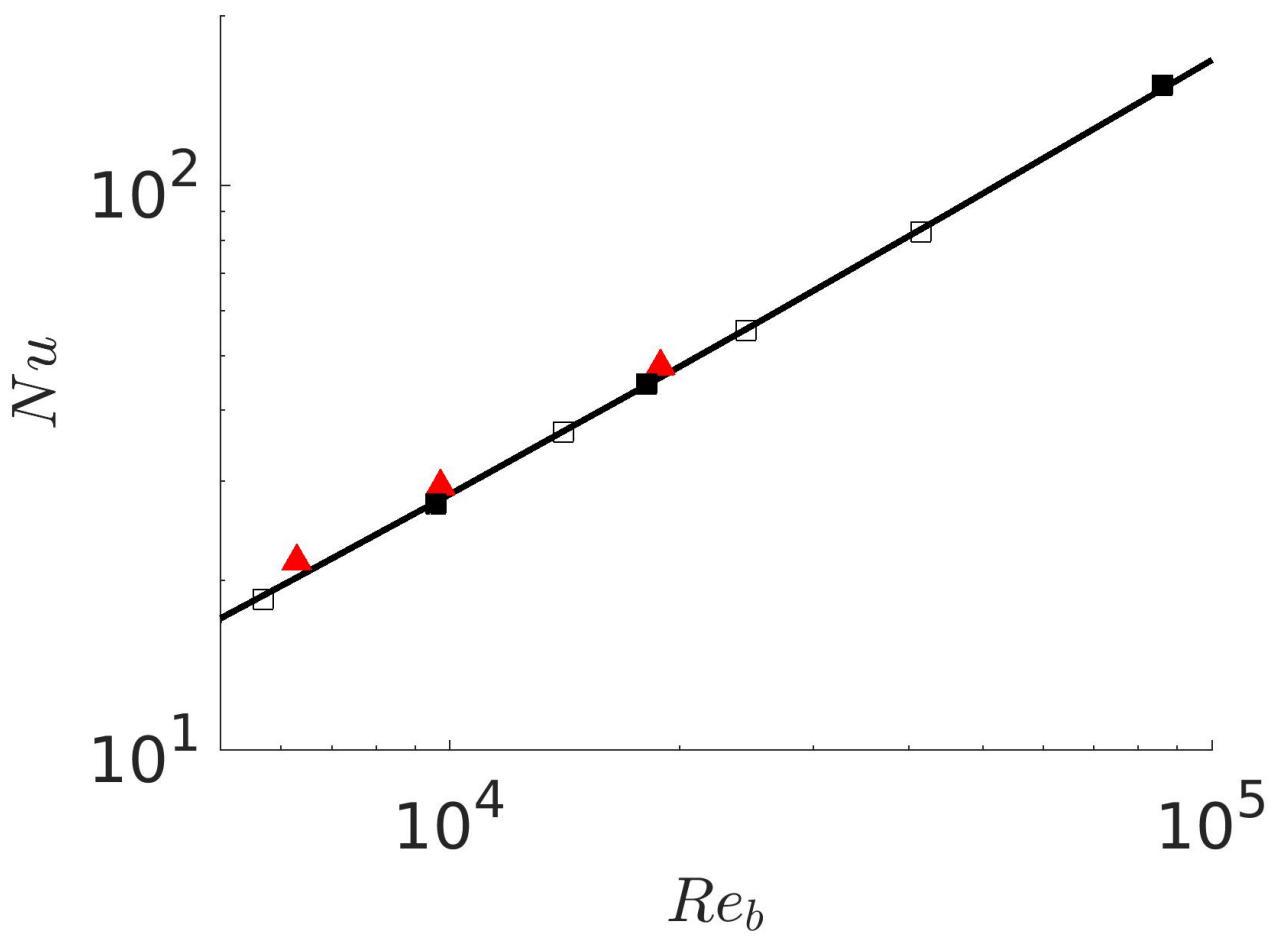

Figure 7: Colour online. Nusselt number as a function of $R e_{b}$ for Prandtl number of 0.71 . Black line represents correlation of Abe and Antonia [35]. $\Delta$ represent $N u$ for CFs, $\square$ are the $N u$ values for PFs from [35] and are the $N u$ values for PFs from [24].

$$
\frac{1}{C_{f}}=1.8 \ln \left(R e_{b} \sqrt{C_{f}}\right)-0.163 .
$$

Figure 7 represents the values of the Nusselt numbers obtained for the

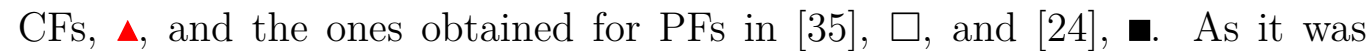
expected, $N u$ for CFs are slightly above the line of the correlation for PFs and when $R e_{b}$ is increased, this difference gets reduced.

A quadrant analysis for case VI, like in [36] for a PF, has been performed to see if the large-scale temperature structures are active in generating wallnormal turbulent heat flux. Also, the generation mechanism of $\overline{v \theta}^{+}$is com- 
pared with the one of $\overline{u v}$ and $\overline{u \theta}^{+}$. For this analysis, the flux is divided in four quadrants. In the case of the wall-normal heat flux, quadrant $1(v>0$ and $\theta>0)$ and quadrant $3(v<0$ and $\theta<0)$, are refered to as the outward and inward interactions. While quadrant $2(v>0$ and $\theta<0)$ and quadrant $4(v<0$ and $\theta>0)$ are refered to as the ejection and sweep events. In Figure 8 all three fluxes are plotted together with the decomposition in the four quadrants. In contrast with the results obtained in [36], the generation mechanism of $\overline{u v}^{+}$and $\overline{v \theta}^{+}$are quite different in a CF. On the other hand, $\overline{u \theta}^{+}$and $\overline{v \theta}^{+}$generation mechanisms are very similar, although interactions and events occur in the opposite walls: for $\overline{u \theta}^{+}$, outward and inward interactions occur in the stationary wall, while for $\overline{v \theta}$, ejection and sweep events happen in this wall. The opposite happens in the moving wall. This is perfectly correlated with the result obtained in the black box of Figure 6b, i.e. the CTFS. In the black box can be seen how, in the lower part of the channel, hot $\theta^{\prime}$ are related with positive $u^{\prime}$ (quadrant 1) and negative $v^{\prime}$ (quadrant 4) or cold $\theta^{\prime}$ are related with negative $u^{\prime}$ (quadrant 3) and positive $v^{\prime}$ (quadrant $2)$. The contrary occurs in the upper part of the channel.

A spectral analysis has been performed for $u^{+}$and $\theta^{+}$. In Figure 9, 1D spectral density of the two intensities is shown for $R e_{\tau}=250$ and 500 , for coordinates $x$ and $z$ and a wall distance of $y^{+}=160$. The most important result is the peak that appears in Figure $9 \mathrm{~b}$ at $\lambda_{z}^{+} \approx 4.7$, which agrees with the one obtained in [37]. The wavelength of the peak corresponds to the width of the CTFS or, in other words, to half of the width of the velocity and thermal fluctuation structures shown previously in Figure $6 \mathrm{~b}$, i.e. $2 L_{R} \approx 4.7$. In addition, it can be appreciated that the peak increases as the Reynolds 


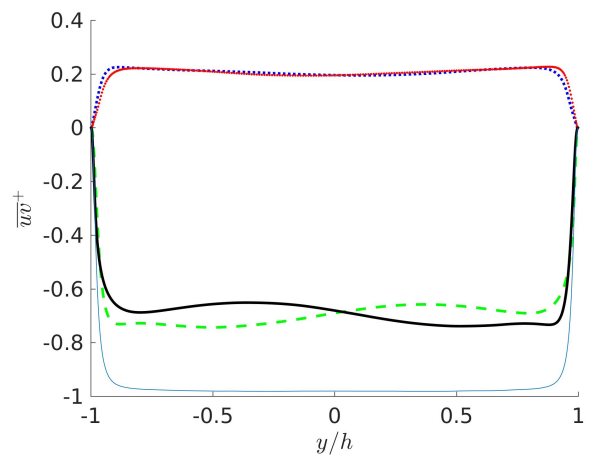

(a)

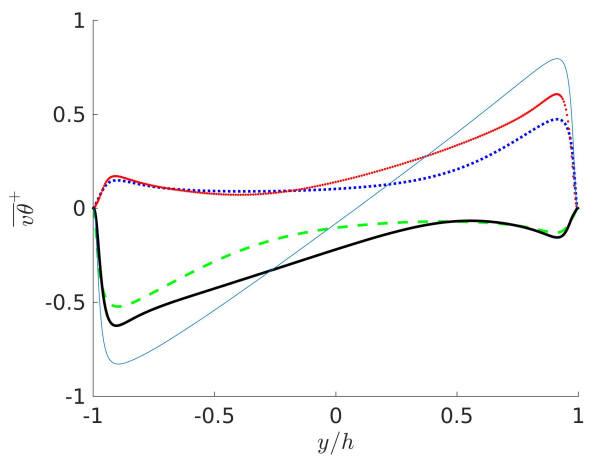

(c)

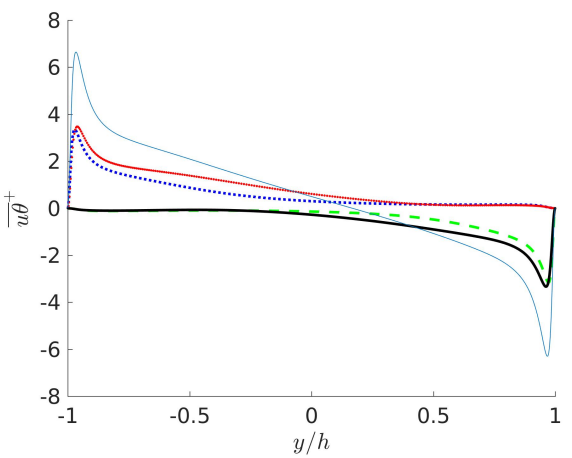

(b)

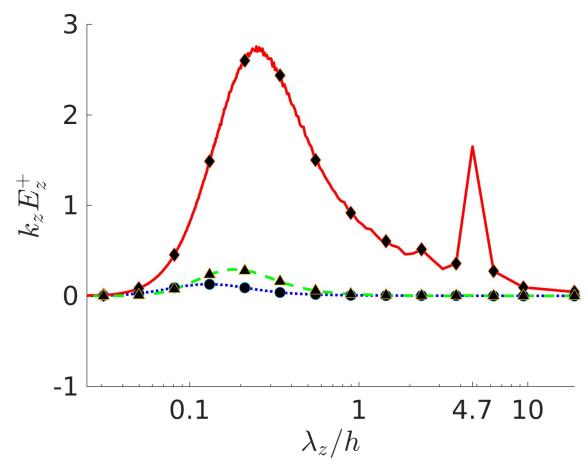

(d)

Figure 8: Colour online. Quadrant analysis of case VI for a) $\overline{u v}^{+}$, b) $\overline{u \theta}^{+}$and c) $\overline{v \theta}^{+}$. Dotted blue line: quadrant 1; dashed green line: quadrant 2; solid red line: quadrant 3; solid black line: quadrant 4 . Solid thin blue line is the sum of the four quadrants. d) 1D coespectral density of $u^{+}, \theta^{+}$and $\overline{u \theta}^{+}$(red solid).

number increases, which means that the structures become more intense for higher Reynolds numbers.

Contour plots are shown in Figure 10 to visualize the $y$ dependences of the spectrum. First, mention that at $y^{+}=10$ there is a concentration of energy corresponding to the small scales of the velocity and temperature, intrinsic to every turbulent flow. The new feature appears at the previously mentioned wavelength, $\lambda_{z}^{+} \approx 4.7$, which corresponds to the CTFS. Here it can 


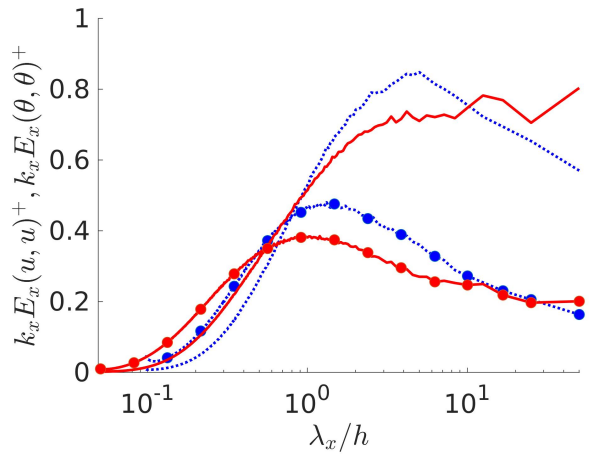

(a)

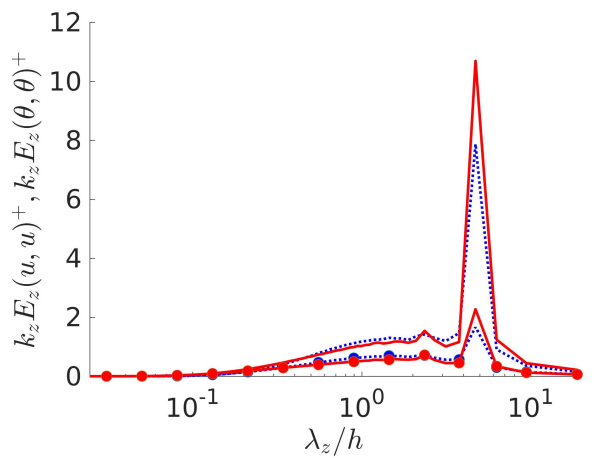

(b)

Figure 9: Colour online. 1D spectral density of $u^{+}$(without marks) and $\theta^{+}(\mathbf{\bullet})$ at $R e_{\tau}=250$ and 500 for (a) $x$ and (b) $z \cdot y^{+}=160$. Colours as in Table 2 .

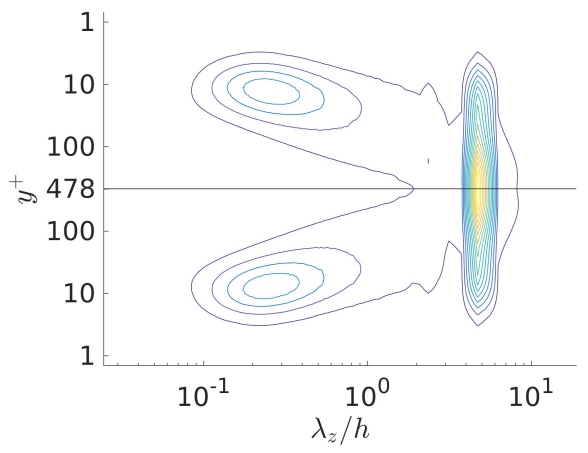

(a)

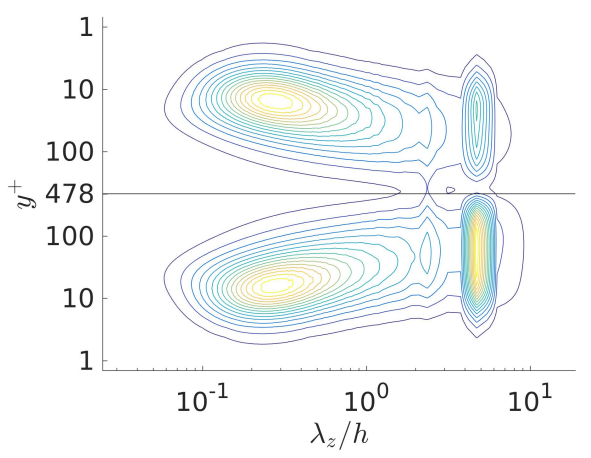

(b)

Figure 10: Colour online. Contours of a) $u^{+}$and b) $\theta^{+}$spectral density for $R e_{\tau}=500$ in the $Y-Z$ plane. Central horizontal line is the center of the channel. Vertical axis is the wall distance to the closest wall. 
As it was said before in Figure $4 \mathrm{c}$, one of the major differences between PFs and CFs, when the same thermal boundary condition is used, is the different behaviour of the streamwise thermal flux, $\overline{u \theta}$, shown in Figure $11 \mathrm{c}$. When the MBC is used, this flux is symmetric in the case of PFs and (almost) antisymmetric for CFs. This antisymmetry is totally related to the second symmetry of the rolls explained above. In the lower half of the channel, hot temperature fluctuations are related to positive velocity fluctuations and vice-versa, causing a positive value of $\overline{u \theta}$. This situation is inverted in the upper part, where hot temperature fluctuations appear with negative velocity fluctuations, causing a negative value of $\overline{u \theta}$. Other point of view to explain the antisymmetry of $\overline{u \theta}$ is the difference in the flow and thermal boundary conditions. In a $\mathrm{CF}$, the flow boundary conditions is that one wall is moving, while other wall is stationary. This is analogous to a temperature difference for the thermal boundary condition. However, in the present work, the MBC is used for the thermal field. Therefore, both walls are kept at the same temperature, which is analogous to a Poiseuille boundary condition for the velocity field.

All intensities related with temperature (Figures 11b, 11c, and 11d) are almost symmetric or antisymmetric, in contrast with PFs, where perfect symmetry or antisymmetry is always present in both halves of the channel. Values of $\theta^{\prime+}$ have been compared with other trusted simulations for PFs [34] and pipe flows [38] and tendencies agree fairly well.

As it was shown before, the effect of the box size in the mean flow, both in the mean temperature or the Nusselt number is small. This is not the case for the statistics of the perturbations. As it can be seen in Figure 11 , 


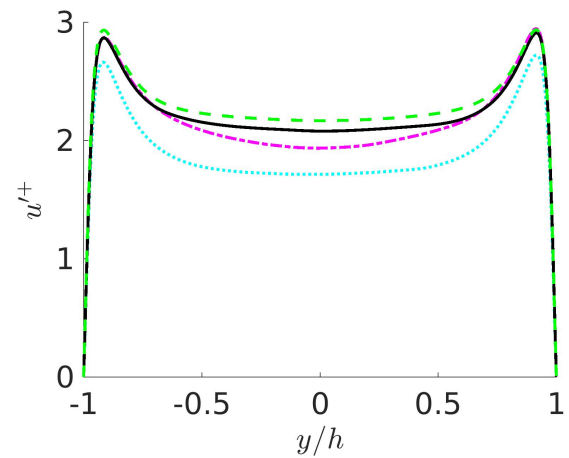

(a)

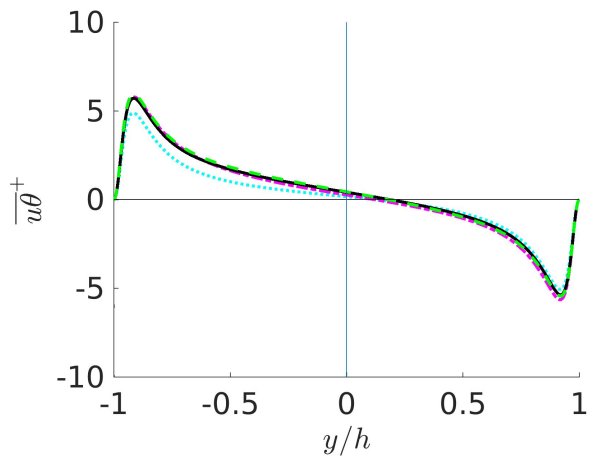

(c)

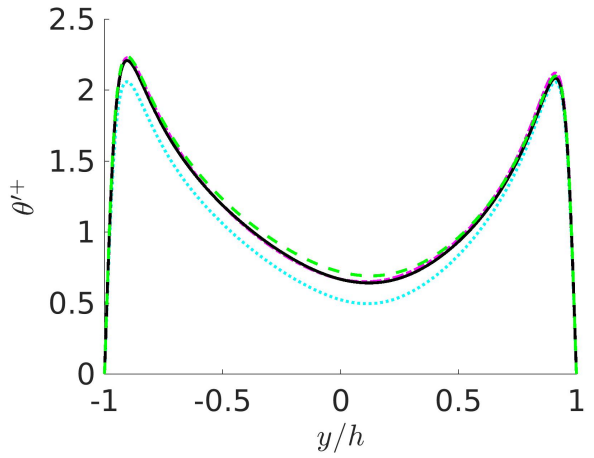

(b)

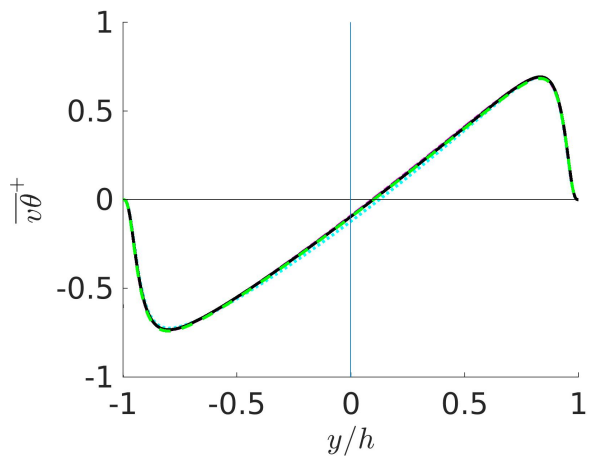

(d)

Figure 11: Colour online. (a) $u^{\prime+}$, (b) $\theta^{\prime+}$, (c) $\overline{u \theta}$ and (d) $\overline{v \theta}$ for cases I-IV. Colours as in Table 2 
the one-point statistics of the flow for $R e_{\tau}=180$ in different boxes do not collapse. The problem is evident in Figures 11a, 11b, and 11c, mainly for case I, but not only. However, in the case of PFs, a $(2 \pi h, \pi h)$ box in $x$ and $z$, respectively, is enough to accurately compute every first-order statistics [26, 27]. The collapsing problem here is also not an effect of the mesh size, as the cell lengths used for cases I-IV are smaller than in many other available DNS.

Thus, the collapse problem has to be related to the CTFS. An analysis of $\theta$ in cases I-IV shows that the CTFS are always present (Figure 12), even if the box is too small (case I) to obtain accurate statistics [26, 27]. The width of the CTFS grows with the box until a transition phase, where a new CTFS fits in the box. For the two smaller computational boxes in the $z$ direction $(\pi / 2$ and $\pi)$, only one CTFS appears (Figure 12 top and middle). Therefore, $L_{R} / h=0.78$ and 1.57 for cases I and II, respectively. However, when extending the computational box to $2 \pi$ in the spanwise direction, a transition phase occurs (Figure 12 bottom). Two velocity vortices and four thermal ones are perfectly visible in the left part of the channel. In the right side, the same CTFS is starting to be created, but it is still in process. A slightly wider computational box is needed in order to properly capture 2 CTFS perfectly structured. This explains the differences in the statistics and the discrepancy about the width of the velocity CTFS with another works [7, 8, 15]. Accurate statistics, both for the flow and the thermal field, need wide boxes, with at least four CTFS well represented. 

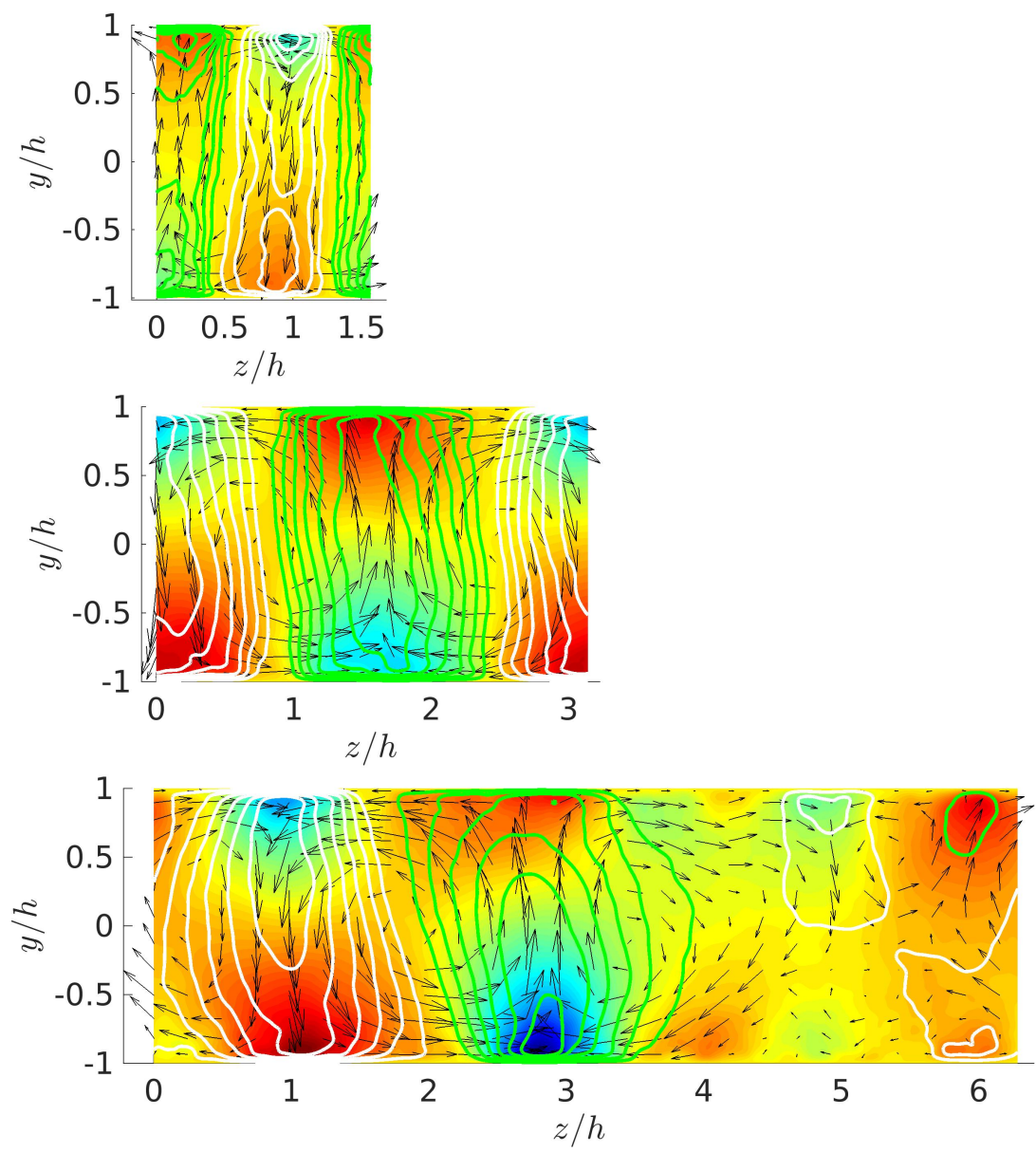

Figure 12: Colour online. Top: case I. Middle: case II. Bottom: case III. Contours of $\langle\theta\rangle_{x}$. White and green lines represent contours of positive and negative $\langle u\rangle_{x}$, respectively. $\left(\langle v\rangle_{x},\langle w\rangle_{x}\right)$ vector field is represented by arrows. 


\section{Summary and conclusions}

In summary, two simulations of thermal flows at larger numbers of Reynolds and larger boxes than those previously simulated have been described. The perturbations of the flow field present a well organized structure in form of positive and negative velocity rolls. These structures leave a trace in the time-averaged flow field. The superstructure made of two velocity rolls and four thermal ones in the time-averaged field has been defined as a Couette Thermal Flow Superstructure. This implies that unlike for the PF, there seems to be less spanwise meandering of large-scale structures in the CF. In this context, Abe et al. [36] also reported in the PF that when the streamwise domain is reduced down to $L_{x} \approx 400$ at $R e_{\tau}=1020$, the two-dimensional behavior is established so that there is a tight coupling between the near-wall and outer-layer structures. This tight coupling may also be observed in the present CTFS and will be studied in future works. The CTFS presents two symmetries and their size and number has been found to depend on the computational box size. Thermal quantities appear to be not totally symmetric or antisymmetric, in contrast to PFs. Using a second set of simulations at $R e_{\tau}=180$, it has been seen that the CTFS do not affect neither the mean velocity nor the mean thermal flow. Moreover, mean-related quantities such as the Nusselt number are also not affected. On the other hand, the width and numbers of rolls, i.e. the size of the computational box, affect the value of the first order statistics of the flow. This explain the small differences found in the bibliography about the width of these rolls. Moreover, this makes the width of the box an essential parameter to obtain accurate statistics. Symmetry-based theories [39], can give an insight into why this CTFS con- 
figuration is totally lost in Poiseuille flows. The statistics of all simulations can be downloaded from the web page of our group.

\section{Acknowledgments}

This work was supported by the MINECO/FEDER, under project ENE201571333-R. The computations of the new simulations were made possible by a generous grant of computing time from the Barcelona Supercomputing Centre, reference FI-2018-1-0037. FAA is partially funded by GVA/FEDER project ACIF2018. We are very grateful for the advices and revision provided by one of the referees of the article, as it has helped to enrich its content.

\section{References}

[1] J. Kim, P. Moin, and R. Moser. Turbulence statistics in fully developed channels flows at low Reynolds numbers. Journal of Fluid Mechanics, 177:133-166, 1987.

[2] Sergio Hoyas and Javier Jiménez. Scaling of the velocity fluctuations in turbulent channels up to $R e_{\tau}=2003$. Physics of Fluids, 18(1):011702, 2006.

[3] M. Bernardini, S. Pirozzoli, and P. Orlandi. Velocity statistics in turbulent channel flow up to $R e_{\tau}=4000$. Journal of Fluid Mechanics, 758:327-343, 2014.

[4] M. Lee and R. Moser. Direct numerical simulation of turbulent channel flow up to $R e_{\tau} \approx 5200$. Journal of Fluid Mechanics, 774:395-415, 2015. 
[5] Yoshinobu Yamamoto and Yoshiyuki Tsuji. Numerical evidence of logarithmic regions in channel flow at $R e_{\tau}=8000$. Physical Review Fluids, 3:012602(R), 2018.

[6] T. Tsukahara, H. Kawamura, and K. Shingai. Dns of turbulent couette flow with emphasis on the large-scale structure in the core region. Journal of Turbulence, 7:1-16, 2006.

[7] V. Avsarkisov, S. Hoyas, M. Oberlack, and J.P. García-Galache. Turbulent plane Couette flow at moderately high reynolds number. Journal of Fluid Mechanics, 751:R1, 2014.

[8] S. Pirozzoli, M. Bernardini, and P. Orlandi. Turbulence statistics in couette flow at high reynolds number. Journal of Fluid Mechanics, 758:323$343,2014$.

[9] M. Lee and R. Moser. Extreme-scale motions in turbulent plane couette flows. Journal of Fluid Mechanics, 842:128-145, 2018.

[10] Sergio Gandía-Barberá, Sergio Hoyas, Martin Oberlack, and Stefanie Kraheberger. The link between the Reynolds shear stress and the large structures of turbulent Couette-Poiseuille flow. Physics of Flu$i d s, 30(4): 041702,2018$.

[11] J. Komminaho, A. Lundbladh, and A. Johansson. Very large structures in plane turbulent Couette flow. Journal of Fluid Mechanics, 320:259258, 1996.

[12] N. Tillmark and P.H. Aldredsson. Large scale structures in turbulent plane couette flow. Advances in Turbulence VII, pages 59-62, 1998. 
[13] O. Kitoh, K. Nakabyashi, and F. Nishimura. Experimental study on mean velocity and turbulence characteristics of plane Couette flow: LowReynolds-number effects and large longitudinal vortical structure. Journal of Fluid Mechanics, 539:199-227, 2005.

[14] O. Kitoh and M. Umeki. Experimental study on large-scale streak structure in the core region of turbulent plane Couette flow. Physics of Fluids, 20(2):025107, 2008.

[15] N. Tillmark. Experiments on transition and turbulence in plane Couette flow. PhD thesis, KTH, Royal Institute of Technology, 1995.

[16] S. Kraheberger, S. Hoyas, and M. Oberlack. Dns of a turbulent couette flow at constant wall transpiration up to $R e_{\tau}=1000$. Journal of Fluid Mechanics, 835:421-443, 2018.

[17] H. Kawamura, H. Abe, and K. Shingai. DNS of turbulence and heat transport in a channel flow with different Reynolds and Prandtl numbers and boundary conditions. Turbulence, Heat and Mass Transfer 3 (Proc. of the 3rd International Symposium on Turbulence, Heat and Mass Transfer), 2000.

[18] B. Debusschere and C.J. Rutland. Turbulent scalar transport mechanisms in plane channel and couette flows. International Journal of Heat and Mass Transfer, 47(8-9):1771-1781, 2004.

[19] T. Tsukahara, H. Kawamura, and K. Shingai. Dns of turbulent heat transfer in plane couette flow with emphasis on the large-scale structure. Thermal Science \&6 Engineering, 13(4):10-11, 2005. 
[20] J. Slotnick, A. Khodadoust, J. Alonso, D. Darmofal, W. Gropp, E. Lurie, and D. Mavriplis. "cfd vision 2030 study: A path to revolutionary computational aerosciences". NASA TECHNICAL REPORT, (218178), 2014.

[21] M. Duponcheel, L. Bricteux, M. Manconi, G. Winckelmans, and Y. Bartosiewicz. Assessment of rans and improved near-wall modeling for forced convection at low prandtl numbers based on les up to $R e_{\tau}=2000$. International Journal of Heat and Mass Transfer, 75:470-482, 2014.

[22] G. Grötzbach. Challenges in low-prandtl number heat transfer simulation and modelling. Nuclear Engineering and Design, 264:41-55, 2013.

[23] S Saha, Cheng Chin, HM Blackburn, and ASH Ooi. The influence of pipe length on thermal statistics computed from DNS of turbulent heat transfer. International Journal of Heat and Fluid Flow, 32(6):1083-1097, 2011.

[24] Alcántara-Ávila, F. and Hoyas, S. and Pérez-Quiles, M.J. DNS of thermal channel flow up to $R e_{\tau}=2000$ for medium to low Prandtl numbers. International Journal of Heat and Mass Transfer, 127:349-361, 2018.

[25] R. Vinuesa, C. Prus, P. Schlatter, and H.M. Nagib. Convergence of numerical simulations of turbulent wall-bounded flows and mean crossflow structure of rectangular ducts. Meccanica, 51(12):3025-3042, 2016.

[26] A. Lozano-Durán and J. Jiménez. Effect of the computational domain on direct simulations of turbulent channels up to $R e_{\tau}=4200$. Physics of Fluids, 26(1):011702, 2014. 
[27] F. Lluesma-Rodríguez, S. Hoyas, and MJ Peréz-Quiles. Influence of the computational domain on dns of turbulent heat transfer up to $R e_{\tau}=$ 2000 for $\operatorname{Pr}=0.71$. International Journal of Heat and Mass Transfer, 122:983-992, 2018.

[28] N Kasagi, Y Tomita, and A Kuroda. Direct numerical simulation of passive scalar field in a turbulent channel flow. Journal of Heat Transfer, 114(3):598-606, August 1992.

[29] S. Hoyas and J. Jiménez. Reynolds number effects on the Reynoldsstress budgets in turbulent channels. Physics of Fluids, 20(10):101511, 2008.

[30] V. Avsarkisov, M. Oberlack, and S. Hoyas. New scaling laws for turbulent Poiseuille flow with wall transpiration. Journal of Fluid Mechanics, 746:99-122, 2014.

[31] S. K. Lele. Compact finite difference schemes with spectral-like resolution. Journal of Computational Physics, 103(1):16-42, 1992.

[32] Philippe R Spalart, Robert D Moser, and Michael M Rogers. Spectral methods for the Navier-Stokes equations with one infinite and two periodic directions. Journal of Computational Physics, 96(2):297-324, 1991.

[33] Hiroyuki Abe, Robert Anthony Antonia, and Toh Sadayoshi. Largescale structures in a turbulent channel fow with a minimal streamwise fow unit. Journal of Fluid Mechanics, 850:733-768, 2018. 
[34] Hiroshi Kawamura, Kouichi Ohsaka, Hiroyuki Abe, and Kiyoshi Yamamoto. DNS of turbulent heat transfer in channel flow with low to medium-high Prandtl number fluid. International Journal of Heat and Fluid Flow, 19(5):482-491, 1998.

[35] Hiroyuki Abe and Robert Anthony Antonia. Relationship between the heat transfer law and the scalar dissipation function in a turbulent channel flow. Journal of Fluid Mechanics, 830:300-325, 2017.

[36] Hiroyuki Abe and Robert Anthony Antonia. Mean temperature calculations in a turbulent channel flow for air andmercury. International Journal of Heat and Mass Transfer, 132:1152-1165, 2019.

[37] S. Hane, T. Tsukahara, and H. Kawamura. DNS of turbulent heat trander in plane Couette flow. Proc of 13th International Heat Transfer Conference, August 2006.

[38] Steffen Straub, Daniel Beck, Bettina Frohnapfel, Philipp Schlatter, and Ricardo Vinuesa. The effect of the selected thermal boundary conditions on a fully developed turbulent pipe flow. 16th European Turbulence Conference, August 2017.

[39] M. Oberlack. A unified approach for symmetries in plane parallel turbulent shear flows. Journal of Fluid Mechanics, 427:299-328, 2001. 